\title{
Absorptive clearance of DTPA as an aerosol-based biomarker in the cystic
} fibrosis airway

\author{
T.E. Corcoran*, K.M. Thomas”, M.M. Myerburg*, A. Muthukrishnan ${ }^{*}$, L. Weber", \\ R. Frizzell ${ }^{+}$and J.M. Pilewski*, ${ }^{*}$
}

ABSTRACT: Biomarkers providing in vivo quantification of the basic elements of cystic fibrosis (CF) lung disease are needed. A study was performed to determine whether the absorption of a small radiolabelled hydrophilic molecule (Indium-111 (In-)DTPA) would be increased in CF airways. DTPA clearance has been used previously to assess epithelial permeability and may also be useful for quantifying liquid absorption.

The absorptive clearance rate of DTPA was quantified in $10 \mathrm{CF}$ and 11 control subjects using a novel aerosol technique. Subjects inhaled an aerosol containing nonabsorbable technetium-99m sulfur colloid (TC-SC) particles and In-DTPA. TC-SC clearance from the lung is exclusively mucociliary, while In-DTPA is cleared by both absorption and mucociliary clearance. The difference between the In-DTPA and Tc-SC clearance rates estimates In-DTPA absorption.

TC-SC (mucociliary) clearance was similar in central and peripheral zones in CF and non-CF lungs. Total In-DTPA clearance was increased in both zones in CF lungs. The absorptive component of In-DTPA clearance was increased in the airway-dominated central lung zones in CF $\left(42 \% \cdot h^{-1}\right.$ versus $\left.32 \% \cdot h^{-1}, p=0.03\right)$.

The absorption of In-DTPA is increased in the CF airway. Further study is needed to understand the relative roles of fluid absorption, inflammation and other mechanisms potentially affecting epithelial permeability and DTPA absorption.

KEYWORDS: Aerosol, clearance, cystic fibrosis, DTPA, epithelial permeability

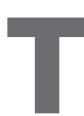
here is a substantial need for new biomarkers in the study of cystic fibrosis (CF) lung disease [1]. Conventional end-points, such as rate of decline in forced expiratory volume in $1 \mathrm{~s}$ (FEV1), require prolonged trials and large sample sizes to demonstrate therapeutic efficacy. Given the small number of CF patients, preliminary efficacy studies are cost-prohibitive, highlighting the need for rapid outcome measures such as biomarkers. Ideally, such biomarkers would provide a quantitative window to the most basic aspects of CF pathophysiology, allowing for the development and evaluation of therapies prior to large-scale clinical trials. The basic defect of CF lung disease occurs in the airways where dysfunction of the CF transmembrane conductance regulator and epithelial sodium channels is thought to create an ionic gradient that causes excessive liquid absorption across the epithelium [2-7]. This results in a dehydrated airway surface liquid layer, defective mucociliary clearance and an increased proclivity for infection and inflammation.

Aerosol-based methods have been developed to measure mucociliary clearance in the lung and used to demonstrate the efficacy of inhaled osmotic therapies [8-10]. We have developed an aerosol technique to measure both mucociliary clearance and the absorptive clearance of a hydrophilic small molecule (DTPA) in whole, central and peripheral lung regions.

We hypothesised that the absorptive clearance of DTPA would occur at an increased rate in the CF airway based on several potential contributory mechanisms. Pro-inflammatory cytokines have been shown to increase airway tight junction permeability, potentially resulting in increased DTPA absorption rates with inflammation [11]. Epithelial damage and denudation have been
AFFILIATIONS

*Division of Pulmonary, Allergy, and Critical Care Medicine, \#Dept of Chemical Engineering,

'Dept of Radiology, and

${ }^{+}$Dept of Cell Biology and Physiology, University of Pittsburgh, Pittsburgh, PA, USA.

CORRESPONDENCE

T.E. Corcoran UPMC MUH NW628

3459 Fifth Avenue

Pittsburgh

PA 15213

USA

E-mail: corcorante@upmc.edu

Received:

April 082009

Accepted after revision:

Aug 172009

First published online:

Aug 282009 
shown to directly increase DTPA absorption [12]. Finally, bulk liquid flux across the epithelium caused by osmotic gradients has been shown to alter DTPA absorption rates [13]. Presumably this occurs through a solvent drag mechanism that affects the amount of DTPA available at the epithelial surface for paracellular absorption. Liquid hyperabsorption after volume addition has been well described in in vitro models of the CF airway [14, 15] and may contribute to increased DTPA absorption rates in the CF airway after aerosol volume addition. Measurements of DTPA absorption in the airways may be useful for detecting the acute changes in liquid absorption that would be expected after successful treatment with an osmotic or a channel-modulating therapy. The same biomarker, if applied longitudinally, might be useful for quantifying the decreases in epithelial permeability that may be expected with an anti-inflammatory therapy or with a healing epithelium.

\section{MATERIALS AND METHODS}

In this pilot study, we compared measurements of mucociliary clearance and DTPA absorptive clearance in whole, central and peripheral lung zones of $10 \mathrm{CF}$ subjects and 11 healthy controls using a dual isotope technique.

\section{Aerosol delivery}

Subjects were enrolled in two sequential groups $(n=11$ : six controls, five $\mathrm{CF}$; $\mathrm{n}=10$ : five controls, five $\mathrm{CF}$ ). Both groups inhaled the test aerosols, which contained technetium-99m sulfur colloid (Tc-SC) and Indium-111 (In-)DTPA in normal saline from a Whisper-Jet nebuliser (Vital Signs Colorado, Inc., Englewood, CO, USA) driven by $8 \mathrm{~L} \cdot \mathrm{min}^{-1}$ oxygen. Group 1 inhaled for $8 \mathrm{~min}$ without delivery controls. Group 2 inhaled for 4 min using visual feedback to control inhalation flow rate $\left(0.5 \mathrm{~L} \cdot \mathrm{s}^{-1}\right)$ and audible feedback to maintain a set respiratory pattern. This change in delivery technique was made to improve inter-subject dosing uniformity. The breathing pattern was intended to provide both central and peripheral lung dosing. Subjects were seated during aerosol delivery. More detail on aerosol delivery is included in the methods supplement, provided as part of the online supplementary material.

\section{Imaging and image analysis}

Subjects were imaged continuously for $60 \mathrm{~min}$ after aerosol delivery while lying recumbent. Independent windows (based on photon energy level) were used to depict the clearance of Tc-99m and In-111. Counts from the clearance images were corrected for decay time, background and isotope spillover, and fit to single exponential curves. These curves were evaluated at $60 \mathrm{~min}$ and those values reported. (Details are included in the online supplementary material.) As In-DTPA can be cleared from the lung by both absorption and mucociliary clearance mechanisms, the absorptive clearance rate was defined as the difference between 60-min clearance rates of In-DTPA and Tc-SC.

Xenon-133 equilibrium images were used to define the lung outlines. Only the right lung was used to avoid interference from the stomach. Posterior images were used. The whole lung region was defined with a lung-shaped outline, while central clearance was measured within a rectangular zone with one-half the height and one-half the width of a rectangle containing the whole lung outline. The peripheral zone was the difference between the whole and central lung zones.

We estimated the radiation exposure associated with aerosol delivery and xenon inhalation to be $\sim 68 \mathrm{mREM}(0.68 \mathrm{mSv})$, effective dose equivalent.

\section{Statistical methods}

Measurements of Tc-SC, In-DTPA total and In-DTPA absorptive clearance were compared by zone in $\mathrm{CF}$ and control subjects using nonparametric (Mann-Whitney) tests. A series of independent linear regressions was performed comparing measurements of aerosol dose and distribution with clearance rates to determine whether differences in aerosol delivery techniques or normal inter-subject dosing variations might have affected the study results. Deposited counts of each isotope in each zone were included in this analysis along with central/peripheral dose ratios for both In-DTPA and Tc-SC (c/ $\mathrm{p}$ In and $\mathrm{c} / \mathrm{p} \mathrm{Tc}$ ).

\section{RESULTS}

\section{Subject characteristics}

Testing groups 1 and 2 were combined for preliminary analysis. Two subjects (one CF, one control) were identified as obvious outliers with whole lung Tc-SC clearance rates more than two SDs above the average of the group: $25 \%$ and $32 \%$, respectively, versus a mean \pm SD of $10 \pm 7 \%$ in the CF group and $9 \pm 9 \%$ in the control group $(n=21)$. These outliers were excluded from further analyses. Subject data are presented in table $1(n=19)$. The CF subjects exhibited well-maintained pulmonary function, as summarised in table 2 with other potentially pertinent clinical information regarding medication history. The ratios of central/peripheral aerosol deposition are also included in table 1. No significant differences in these ratios were observed when comparing $\mathrm{CF}$ and control subjects (c/p Tc: $p=0.62, c / p$ In: $p=0.41$; Mann-Whitney). Values of c/ $\mathrm{p}$ In were slightly increased compared with $\mathrm{c} / \mathrm{p}$ Tc in 15 out of 19 subjects $(p=0.004$, Wilcoxon signed-rank test).

\begin{tabular}{|c|c|c|}
\hline \multirow[t]{2}{*}{ TABLE 1} & \multirow[b]{2}{*}{ CF subjects } & /peripheral \\
\hline & & Controls \\
\hline Subjects $n$ & 9 & 10 \\
\hline Sequential group $1 \mathrm{n}$ & 5 & 5 \\
\hline Sequential group 2 n & 4 & 5 \\
\hline FEV $1 \%$ pred & $86 \pm 15$ & $99 \pm 8$ \\
\hline $\mathrm{FEV}_{1} \%$ pred $\min -\max$ & $57-104$ & 86-107 \\
\hline FVC \% pred & $95 \pm 9$ & $98 \pm 10$ \\
\hline FEF $25-75 \% \%$ pred & $70 \pm 30$ & $103 \pm 18$ \\
\hline Age yrs & $28 \pm 12$ & $30 \pm 7$ \\
\hline $\mathrm{c} / \mathrm{p}$ ratio $\mathrm{Tc}-\mathrm{SC}$ & $0.64 \pm 0.17$ & $0.59 \pm 0.16$ \\
\hline$c / p$ ratio In-DTPA & $0.73 \pm 0.22$ & $0.63 \pm 0.20$ \\
\hline \multicolumn{3}{|c|}{$\begin{array}{l}\text { Data are presented as mean } \pm \mathrm{SD} \text {, unless otherwise stated. CF: cystic fibrosis; } \\
\text { FEV1: forced expiratory volume in } 1 \mathrm{~s} \text {; \% pred: \% predicted; FVC: forced vital } \\
\text { capacity; FEF25-75\%: forced expiratory flow at } 25-75 \% \text { of FVC; Tc-SC: } \\
\text { technetium-99m sulfur colloid; In: indium. Comparing c/p ratios in CF versus } \\
\text { controls: c/p Tc-SC } p=0.62 ; c / p \text { In-DTPA: } p=0.41 \text { (Mann-Whitney). }\end{array}$} \\
\hline
\end{tabular}




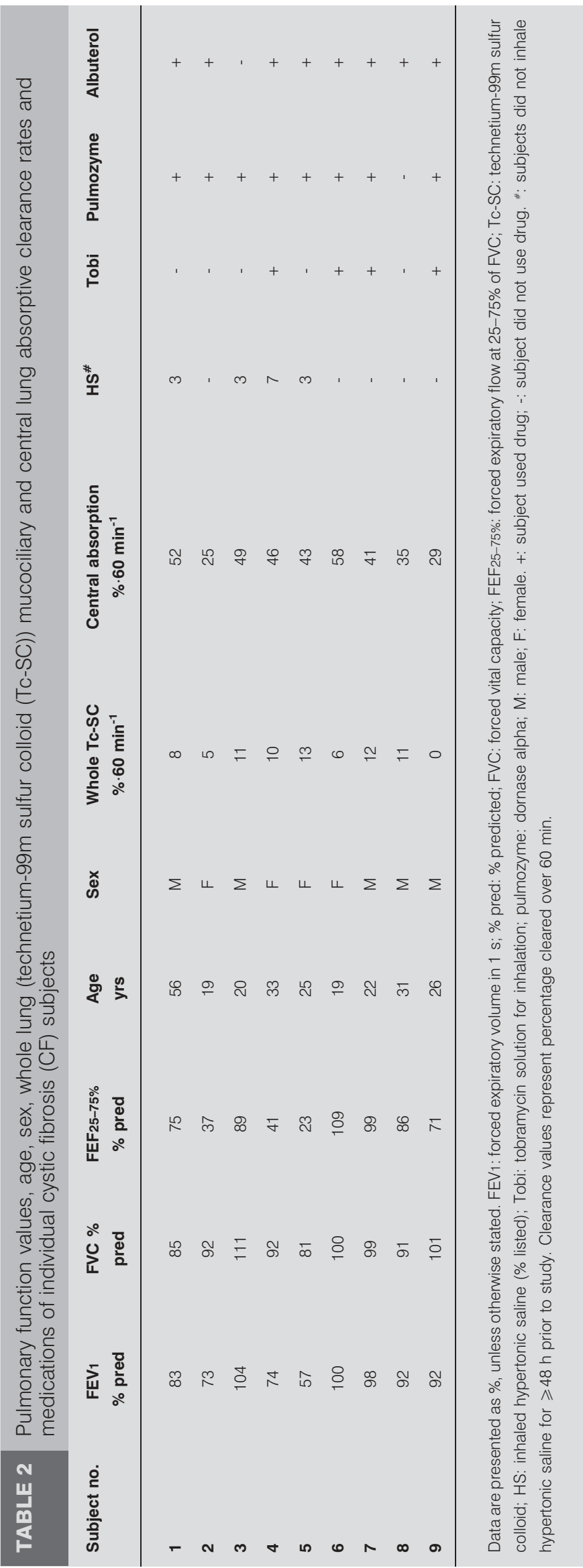

\section{Mucociliary (TC-SC) clearance rates are similar in CF and control groups}

Average clearance rates for Tc-SC and In-DTPA are reported by zone in table 3. Mucociliary (Tc-SC) clearance rates were similar in CF and control subjects in whole, central and peripheral lung zones as shown in figure 1.

\section{Total In-DTPA clearance rates are increased in CF}

In contrast to measurements of Tc-SC clearance, clearance rates of In-DTPA were significantly increased in CF subjects in all zones, as shown in table 3 and figure 2. These total InDTPA clearance rates, which include both mucociliary and absorptive components, were similar within the groups across the central and peripheral lung zones.

\section{Absorptive clearance rates are increased in whole and central zones in $\mathbf{C F}$}

The absorptive component of In-DTPA clearance, defined as the In-DTPA clearance rate minus the Tc-SC clearance rate, was significantly increased in CF in whole and central zones, as shown in table 3 and figure 3. There was a trend towards increased absorptive clearance in the peripheral zones in $\mathrm{CF}$, but the difference was not statistically significant $(p=0.14)$.

\section{Effect of outliers}

Inclusion of the two outliers did not affect any previously reported trends but did reduce overall statistical significance. When the outliers were included $(n=21)$, the mean \pm SD whole lung In-DTPA clearance rate was $55 \pm 14 \% \cdot h^{-1}$ in CF versus $45 \pm 8 \% \cdot \mathrm{h}^{-1}$ in controls $(\mathrm{p}=0.09)$. Mean central lung In-DTPA clearance was $55 \pm 16 \% \cdot \mathrm{h}^{-1}$ in CF versus $44 \pm 10 \% \cdot \mathrm{h}^{-1}$ in controls $(p=0.07)$, and the mean peripheral lung In-DTPA clearance rate was $55 \pm 13 \% \cdot h^{-1}$ in CF versus $46 \pm 9 \% \cdot h^{-1}$ in controls $(p=0.08)$. The mean whole lung absorptive clearance rate was $45 \pm 15 \% \cdot h^{-1}$ in CF versus $36 \pm 8 \% \cdot h^{-1}$ in controls $(p=0.09)$.

TABLE 3 Measurements of technetium-99m sulfur colloid
(Tc-SC) and total indium-111 (In-)DTPA
clearance, and the absorptive component of
In-DTPA clearance in cystic fibrosis (CF) and
control subjects

\begin{tabular}{lccc} 
& CF & Controls & p-values \\
\hline Subjects $\mathbf{n}$ & 9 & 10 & \\
$\begin{array}{l}\text { Tc-SC (mucociliary) } \\
\quad \text { Whole lung clearance }\end{array}$ & & \\
$\quad$ Central lung clearance & $8 \pm 4$ & $7 \pm 4$ & 0.41 \\
$\quad$ Peripheral lung clearance & $14 \pm 12$ & $11 \pm 7$ & 0.51 \\
In-DTPA & $6 \pm 7$ & $4 \pm 5$ & 0.62 \\
$\quad$ Whole lung clearance & & & \\
Central lung clearance & $56 \pm 14$ & $44 \pm 8$ & 0.04 \\
Peripheral lung clearance & $56 \pm 16$ & $43 \pm 9$ & 0.05 \\
Absorptive whole lung clearance & $56 \pm 13$ & $44 \pm 8$ & 0.04 \\
Absorptive central lung clearance & $48 \pm 12$ & $37 \pm 8$ & 0.04 \\
Absorptive peripheral lung clearance & $42 \pm 11$ & $32 \pm 7$ & 0.03 \\
& $50 \pm 13$ & $40 \pm 9$ & 0.14 \\
\hline
\end{tabular}

Data are reported as percentage cleared over $60 \mathrm{~min}$. Data are presented as mean $\pm S D$, unless otherwise stated. All p-values achieved using a MannWhitney test. 


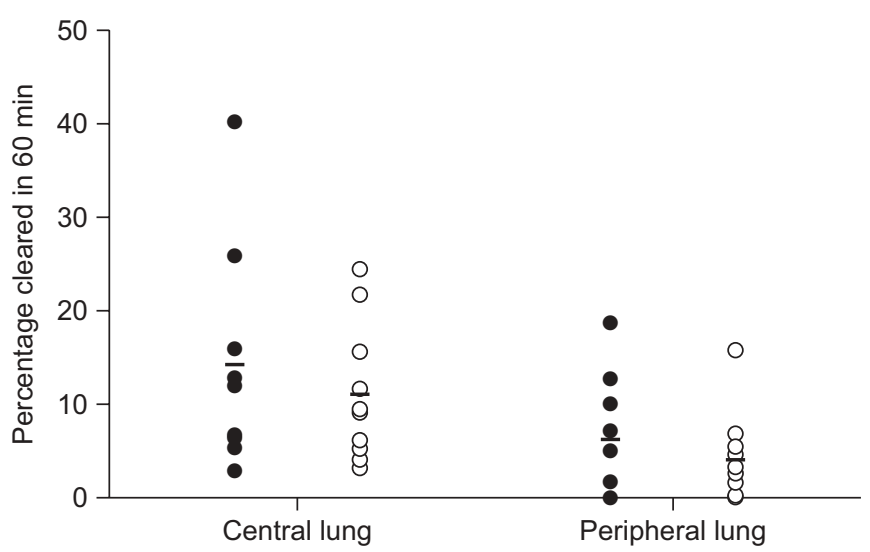

FIGURE 1. Central and peripheral lung clearance rates of technetium-99m sulfur colloid (Tc-SC) in cystic fibrosis $(\bullet ; n=9)$ and control $(O ; n=10)$ subjects. This represents the rate of mucociliary clearance. Data are reported as percentage cleared over $60 \mathrm{~min}$. Central lung: $p=0.51$, peripheral lung: $p=0.62$ (Mann-Whitney test).

Mean central lung absorptive clearance was $39 \pm 15 \% \cdot \mathrm{h}^{-1}$ in CF versus $30 \pm 9 \% \cdot h^{-1}$ in controls $(p=0.07)$, and the mean peripheral lung absorptive clearance rate was $47 \pm 15 \% \cdot \mathrm{h}^{-1}$ in $\mathrm{CF}$ versus $40 \pm 8 \% \cdot \mathrm{h}^{-1}$ in controls $(\mathrm{p}=0.23)$.

\section{Linear regression modelling to determine the effects of dosing variability}

Linear regression was used to determine whether differences in deposited dose caused by aerosol delivery technique or normal inter-subject differences might have affected measurements of clearance. No significant correlations were found between Tc-SC clearance and any dosing variable. $\mathrm{c} / \mathrm{p}$ dose ratios were not predictive of In-DTPA or Tc-SC clearance. The deposited dose of In-DTPA significantly predicted absorptive clearance rate in all zones and total In-DTPA clearance in whole and peripheral zones. The absorptive clearance rate was inversely related to In-DTPA dose with higher doses clearing more slowly on a percentage per hour basis. Central lung absorptive clearance was significantly increased in CF subjects compared with controls using a linear regression model that included the effects of deposited In-DTPA dose $(p=0.05)$.

\section{DISCUSSION}

Mucociliary clearance plays a vital host defence role in the lungs by providing rapid mechanical clearance of deposited particulates and pathogens. In the current study, no deficits in mucociliary clearance rate were detected in the CF subjects. DONALDSON et al. [9] demonstrated deficits in 24-h clearance in CF that were not detectable in similar 60-min measurements. Both studies included CF groups with well-preserved pulmonary function. These studies imply that baseline deficits in mucociliary clearance may not be detectable in more healthy CF subject groups, at least not using 60-min measurements.

In contrast, In-DTPA clearance was significantly increased in $\mathrm{CF}$ in all lung zones. It was notably rapid, with $56 \%$ of deposited material being cleared within $1 \mathrm{~h}$ in $\mathrm{CF}$ and $44 \%$ in the controls. This clearance includes both mucociliary and absorptive components which can be differentiated through our technique. Absorption was the dominant component,

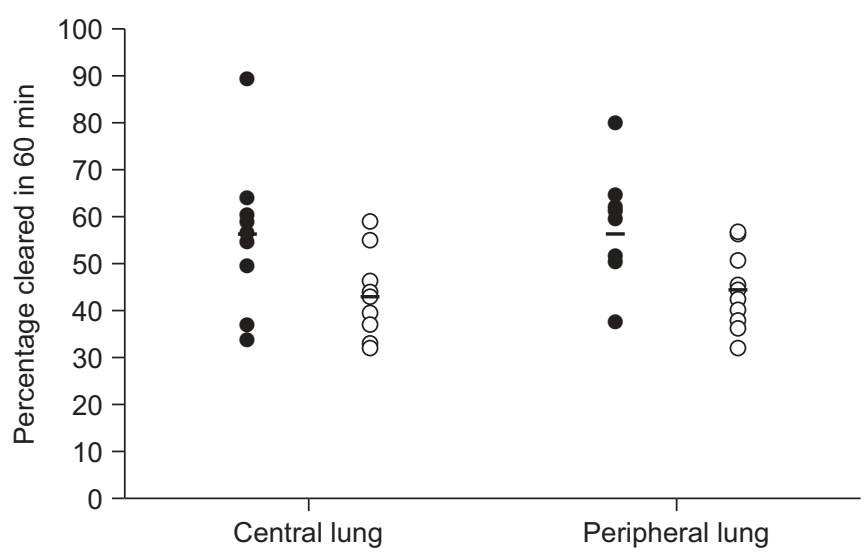

FIGURE 2. Central and peripheral lung clearance rates of Indium-111 (In-) DTPA in cystic fibrosis $(\bullet ; n=9)$ and control $(O ; n=10)$ subjects. In-DTPA is cleared through both adsorptive and mucociliary routes. Data are reported as percentage cleared over $60 \mathrm{~min}$. Central lung: $p=0.05$; peripheral lung: $p=0.04$ (Mann-Whitney test).

comprising $86 \%$ of total whole lung clearance in CF and $84 \%$ in controls. Absorptive clearance was significantly increased in the airway-dominated central lung zones in $\mathrm{CF}$ and also in the whole lung zones.

Whole lung DTPA absorption has been previously used as a gauge of alveolar epithelial permeability [16-19]. In the CF airways, epithelial injury and increases in tight junction permeability associated with infection and inflammation would be expected to increase baseline permeability [11, 12]. DTPA is a small hydrophilic molecule, and it is likely that it remains in an aqueous solution and moves in parallel to fluid flux in the airway. In vivo animal data support the notion that DTPA is a marker of fluid flux. DTPA absorption across an isolated trachea was accelerated or slowed when the airway lumen was bathed with hypotonic or hypertonic fluid (saline or mannitol), respectively [13]. Numerous studies have

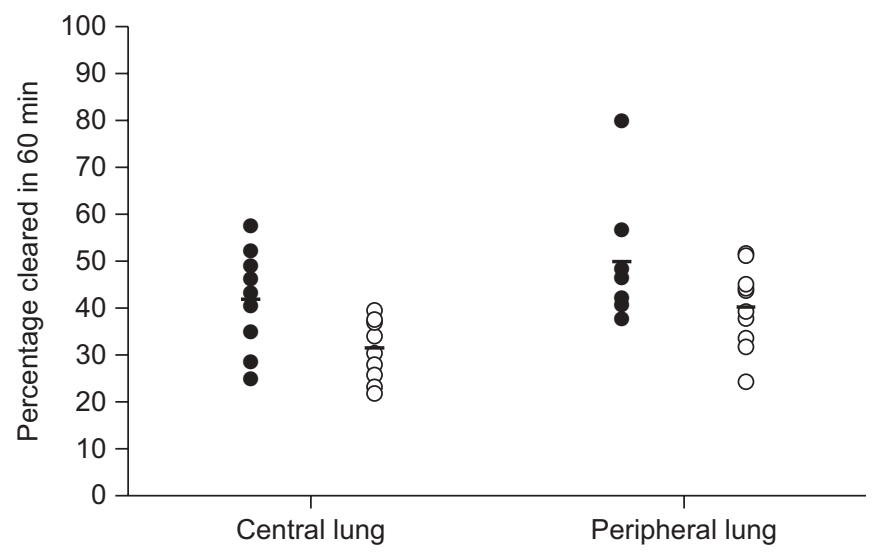

FIGURE 3. Absorptive component of indium-111 (In-)DTPA clearance in central and peripheral lung zones in cystic fibrosis $(\bullet ; n=9)$ and control $(O ; n=10)$ subjects. Absorptive clearance is calculated as the difference between the total InDTPA clearance rate and the technetium-99m sulfur colloid (mucociliary) clearance rate in each subject. Data are reported as percentage cleared over $60 \mathrm{~min}$. Central lung $p=0.03$; peripheral lung $p=0.14$ (Mann-Whitney test). 
demonstrated excessive airway surface liquid absorption in the CF airway [14, 15], and the hyperabsorption of the liquid associated with the aerosol could provide the mechanism for the increased rates of DTPA absorption noted in the CF subjects.

The data from our pilot clinical study do not allow us to differentiate liquid absorptive effects versus effects associated with epithelial permeability. Measurements of DTPA absorption in the airways may be useful for detecting both immediate changes in liquid absorption, as might be found after treatment with an osmotic or a channel-modulating therapy, and longer term changes in epithelial permeability as might be found after anti-inflammatory treatments or with a healing epithelium. Longer term effects would logically require longitudinal measurements. Both of these applications require further study with the next logical step being assessment of response to a known therapy.

Differences in aerosol deposition pattern have been shown to affect measurements of mucociliary clearance [8]. In the present study, two different aerosol delivery techniques were used (details included in the methods provided as part of the online supplementary material). In any study a certain amount of dosing variability can be expected, and when comparing subjects with lung disease and controls, true dose matching is even less likely. Differences in dosing can be accurately depicted through a series of variables considering radioactive counts of each isotope in each lung zone, and the ratio of these doses in central versus peripheral zones. The use of linear regression models provided us with a means to determine and correct for differences in aerosol deposition, ultimately allowing us to combine subject groups receiving the aerosols through different techniques. These regression models demonstrated an important inverse relationship between deposited In-DTPA dose and absorption rate. Higher deposited doses of In-DTPA resulted in proportionally slower percentage per hour absorptive clearance rates in all zones, surprisingly indicating a finite capacity for DTPA transport even across the large surface area of the peripheral lung. After correction for dosing effects, significant increases in central lung absorptive clearance were still noted in the CF subjects.

One limitation of our technique is our inability to truly segregate alveolar and airway zones. Results from the central zones were undoubtedly influenced by alveolar tissue overlying the airways. However, comparisons of the central and peripheral zones are still useful for demonstrating the relative effects of the airways. A comparison of the central and peripheral zones used in our analysis demonstrated expected effects. Mucociliary clearance was 2-3 times higher in the central zones while absorptive clearance was significantly increased in peripheral zones, giving some indication that our designated zones are anatomically distinct. Another study limitation was the presence of two outliers which were removed from the analyses based on whole lung mucociliary clearance measurements that were $>2$ SD above the mean for the group. We believe that technique-related issues (i.e. the inadvertent counting of oesophageal material) must have produced the errant results, although this could not be absolutely determined, and these outliers may indicate that a higher level of variability would be found in a larger population.
This study demonstrates a basic physiological difference in the CF airway that is easily measurable at a macroscopic level, apparent in subjects with well-maintained pulmonary function, and likely to be related to the basic defects of CF lung disease. Increased airway epithelial permeability may contribute to the increased absorption rates demonstrated in the CF subjects. Liquid hyperabsorption may also contribute at a significant level. We believe that our aerosol technique can be further developed into a biomarker to independently quantify either of these mechanisms, providing indication of therapeutic efficacy ahead of currently available indicators. Importantly, these studies can be performed with low to minimal radiation exposures making multiple measurements and application in paediatric populations feasible.

\section{SUPPORT STATEMENT}

The present study was funded by NIH K25HL081533 and P30DK072506, and the Cystic Fibrosis Foundation Research Development Program.

\section{CLINICAL TRIAL}

The present study is a registered clinical trial at ClinicalTrials.gov (NCT00248755 and NCT00541190).

\section{STATEMENT OF INTEREST}

None declared.

\section{ACKNOWLEDGEMENTS}

The authors would like to thank D. Rowlands for his thoughtful review of the manuscript.

\section{REFERENCES}

1 Mayer-Hamblett N, Ramsey BW, Kronmal RA. Advancing outcome measures for the new era of drug development in cystic fibrosis. Proc Am Thorac Soc 2007; 4: 370-377.

2 Hirsh AJ. Altering airway surface liquid volume: inhalation therapy with amiloride and hyperosmotic agents. Adv Drug Deliv Rev 2002; 54: 1445-1462.

3 Knowles MR, Boucher RC. Mucus clearance as a primary innate defense mechanism for mammalian airways. J Clin Invest 2002; 109: 571-577.

4 Mall M, Grubb BR, Harkema JR, et al. Increased airway epithelial $\mathrm{Na}+$ absorption produces cystic fibrosis-like lung disease in mice. Nat Med 2004; 10: 487-493.

5 Matsui H, Grubb BR, Tarran R, et al. Evidence for periciliary liquid layer depletion, not abnormal ion composition, in the pathogenesis of cystic fibrosis airways disease. Cell 1998; 95: 1005-1015.

6 Frizzell RA, Pilewski JM. Finally, mice with CF lung disease. Nat Med 2004; 10: 452-454.

7 Pilewski JM, Frizzell RA. Role of CFTR in airway disease. Physiol Rev 1999; 79: S215-S255.

8 Donaldson SH, Corcoran TE, Laube BL, et al. Mucociliary clearance as an outcome measure for cystic fibrosis clinical research. Proc Am Thorac Soc 2007; 4: 399-405.

9 Donaldson SH, Bennett WD, Zeman KL, et al. Mucus clearance and lung function in cystic fibrosis with hypertonic saline. $N$ Engl J Med 2006; 354: 241-250.

10 Robinson M, Daviskas E, Eberl S, et al. The effect of inhaled mannitol on bronchial mucus clearance in cystic fibrosis patients: a pilot study. Eur Respir J 1999; 14: 678-685.

11 Coyne CB, Vanhook MK, Gambling TM, et al. Regulation of airway tight junctions by proinflammatory cytokines. Mol Biol Cell 2002; 13: 3218-3234. 
12 Wells UM, Woods AJ, Hanafi Z, et al. Tracheal epithelial damage alters tracer fluxes and effects of tracheal osmolaity in sheep in vivo. J Appl Physiol 1995; 78: 1921-1930.

13 Wells UM, Hanafi Z, Widdicombe JG. Osmolality alters tracheal blood flow and tracer uptake in anesthetized sheep. J Appl Physiol 1994; 77: 2400-2407.

14 Chambers LA, Rollins BM, Tarran R. Liquid movement across the surface epithelium of large airways. Respir Physiol Neurobiol 2007; 159: 256-270.

15 Blouquit S, Regnier A, Dannhoffer L, et al. Ion and fluid transport properties of small airways in cystic fibrosis. Am J Respir Crit Care Med 2006; 174: 299-305.
16 Brown MA, Lantz RC, Sobonya R, et al. Aerosolized lipopolysaccharide increases pulmonary clearance of $99 \mathrm{mTc}$-DTPA in rabbits. Am Rev Respir Dis 1992; 146: 1462-1468.

17 Coates G, O'Brodovich H, Dolovich M. Lung clearance of 99mTcDTPA in patients with acute lung injury and pulmonary edema. J Thorac Imaging 1988; 3: 21-27.

18 Groth S. Pulmonary clearance of 99mTc-DTPA. An index of alveolar epithelial permeability. Dan Med Bull 1991; 38: 189-203.

19 Mason GR, Peters AM, Bagdades E, et al. Evaluation of pulmonary alveolar epithelial integrity by the detection of restriction to diffusion of hydrophilic solutes of different molecular sizes. Clin Sci (Lond) 2001; 100: 231-236. 